\title{
Probing nonunitary neutrino mixing via long-baseline neutrino oscillation experiments based at J-PARC
}

\author{
Soumya C.๑* \\ Institute of Physics Bhubaneswar, Sainik School POST, Sachivalaya Marg, 751005 Odisha, India
}

(Received 22 October 2021; accepted 13 December 2021; published 11 January 2022)

\begin{abstract}
This paper investigates the capability of long-baseline experiments, which are making use of neutrinos that are coming from Japan Proton Accelerator Research Complex, in establishing the unitarity of activeneutrino mixing by ruling out the nonunitary mixing scheme as a function of true values of $C P$-violating phase $\delta_{C P}$. It is found that T2HK can establish the unitarity of active neutrino mixing at above $2 \sigma$ C.L. irrespective of neutrino mass hierarchy and the true value of $\delta_{C P}$, if the nonunitary (NU) parameter $\alpha_{21}$ is of the order of $10^{-2}$. Further, this paper also discusses the bound on the NU parameter in the 21 sector and the sensitivity limit of these experiments in determining the NU parameter. It is found that the bounds on $\left(\alpha_{21} / 2\right)$ are $0.028,0.0026,0.005$ at $2 \sigma$ C.L., respectively, for T2K, T2HK, and T2HKK. Moreover, it is also found that the sensitivity limit of T2HK on NU parameter is far better than that of both T2HKK and T2K.
\end{abstract}

DOI: 10.1103/PhysRevD.105.015012

\section{INTRODUCTION}

Ever since the phenomenon of flavor transition of neutrino [1-7] has become one of the center attentions in particle physics, long-baseline (LBL) experiments have played a significant role to understand the hidden nature of the fundamental particle neutrino. With the confirmation of a nonzero reactor mixing angle by both accelerator and reactor neutrino experiments, the three flavor neutrino oscillation paradigm that is governed by two mass squared differences $\left(\Delta m_{21}^{2}\right.$ and $\left.\Delta m_{31}^{2}\right)$ has become the most accepted theoretical model for the neutrino flavor transition. The unitary mixing of active neutrinos $\left(\nu_{e}, \nu_{\mu}\right.$, and $\left.\nu_{\tau}\right)$ in this model is described by three mixing angles $\left(\theta_{12}, \theta_{13}\right.$, $\theta_{23}$ ) and one phase $\delta_{C P}$. Though the oscillation parameters in this paradigm are determined with an unprecedented accuracy, the information about $C P$-violating phase, hierarchy of neutrino masses, and octant of $\theta_{23}$ (i.e., whether $\theta_{23}$ is greater or lesser than $45^{\circ}$ ) are not known. The determination of these unknowns by currently running LBL experiments $(\mathrm{T} 2 \mathrm{~K}$ and $\mathrm{NO} \nu \mathrm{A})$ is quite challenging as there exists degeneracies among the oscillation parameters, and the data collected so far are not sufficient enough to resolve the degeneracies among the parameters. The phase II runs of current generation experiments along with future generation LBL experiments with greater energy resolution,

\footnotetext{
*soumyac20@gmail.com
}

Published by the American Physical Society under the terms of the Creative Commons Attribution 4.0 International license. Further distribution of this work must maintain attribution to the author(s) and the published article's title, journal citation, and DOI. Funded by SCOAP. improved statistics, and magnificent matter effect like the Deep Underground Neutrino Experiment (DUNE), Tokai to Hyper-Kamioka (T2HK), Tokai to Hyper-Kamioka to Korea (T2HKK), and European Spallation Source Neutrino Super Beam (ESS $\nu \mathrm{SB})$ etc., are expected to shed light on remaining unknowns in the neutrino sector.

The discovery of neutrino oscillation not only opens up a way to probe the properties of neutrinos, but it also motivates us to explore physics beyond the Standard Model (SM) as it clearly indicates that the neutrino has a nonzero tiny mass, which is one of the shortcomings of the SM. Consequently, many viable mechanisms collectively known as seesaw mechanisms [8-17] are introduced in the literature to explain the lightness of neutrino mass. However, the models based on low-scale seesaw [18] are more captivating over the high-scale seesaw as these models require strong evidence to support them and within the experimental limit it is difficult to probe high-scale seesaw even using Large Hadron Collider experiments. Whereas in low-scale seesaw models for instance, inverse seesaw [19-21], linear seesaw etc., contain new neutrino states, which do not have any SM interactions, the so-called sterile neutrinos with a mass of the order of a GeV/TeV scale as the seesaw breaking scale in these models can be brought down to $\mathrm{TeV} / \mathrm{GeV}$ scale. Therefore, signatures of such sterile neutrinos can be probed at LHC experiments. Moreover, existence of such sterile neutrinos and their mixing with active neutrinos leads to the nonunitary mixing of active neutrinos. As a result, one can also probe them at long-baseline neutrino experiments by looking at the deviation from the unitary mixing of active neutrinos.

Enormous studies regarding the nonunitary neutrino mixing have been already discussed in the literature in 
both phenomenological and theoretical point of views [22-32]. In [33], it has been shown that nonunitary effects originated from a minimal inverse seesaw model can be probed at neutrino factory experiment. The bounds on nonunitary mixing parameters are obtained in [34,35]. Moreover, the impact of nonunitarity mixing on the determination of various unknowns in neutrino sector such as neutrino mass hierarchy, octant of atmospheric mixing angle, and $C P$-violating phase by long-baseline experiments are discussed in [36-39]. A combined analysis of short and long-baseline neutrino oscillation data in nonunitary mixing scenario has been explored in [40], and it is found that there is no significant deviation from unitary mixing. The results of a combined analysis in neutrino oscillations without unitarity assumption in the three flavor mixing is presented in [41]. In [42], it is found that with the next-generation experimental data, the normalizations of all rows and columns of the lepton mixing matrix will be constrained to $\leq 10 \%$ precision, with the $e$ row best measured at $\leq 1 \%$ and the $\tau$ row worst measured at $\approx 10 \%$ precision. A recent study on nonunitary mixing using current generation experiments showed that the stronger tension that exists between the latest 2020 data of the T2K and $\mathrm{NO} \nu \mathrm{A}$ experiments gets reduced with nonunitary analysis [43]. Another study that obtained the constraints for nonunitarity coming from the observables: the neutrinoantineutrino gamma process and the invisible $Z$ boson decay into neutrinos is presented in [44]. This paper address three basic questions regarding the nonunitary mixing for the first time, which are

(i) Whether the long-baseline experiments based at J-PARC are capable of establishing the unitarity of active neutrino mixing matrix by ruling out nonunitary mixing or not?

(ii) What are the bounds on the nonunitary mixing parameter that can be achieved by these experiments?

(iii) What is the sensitivity limits of these experiments in determining the nonunitary mixing parameter?

This paper is organized as follows. The neutrino oscillation in presence of nonunitary mixing scheme is discussed in Sec. II. Section III discusses the simulation details of the experiments that are considered for this study. The capability of these experiment in establishing unitarity of active neutrino mixing, the bounds on the NU parameter and the sensitivity limits of these experiment in determining NU parameter are, respectively, discussed in Sec. IV. Finally, the summary and conclusions of this study is given in Sec. V.

\section{NEUTRINO OSCILLATION IN PRESENCE OF NONUNITARITY MIXING}

The general form of a unitary neutrino mixing matrix in a model with $n$ sterile neutrinos can be written as

$$
\begin{aligned}
& \mathcal{U}=\left(\begin{array}{cccccc}
U_{e 1} & U_{e 2} & U_{e 3} & \cdot & . & U_{e(n+3)} \\
U_{\mu 1} & U_{\mu 2} & U_{\mu 3} & \cdot & . & U_{\mu(n+3)} \\
U_{\tau 1} & U_{\tau 2} & U_{\tau 3} & \cdot & \cdot & U_{\tau(n+3)} \\
U_{s^{1} 1} & U_{s^{1} 2} & U_{s^{1} 3} & \cdot & \cdot & U_{s^{1}(n+3)} \\
\cdot & \cdot & \cdot & \cdot & \cdot & \cdot \\
\cdot & \cdot & \cdot & \cdot & \cdot & \cdot \\
U_{s^{n} 1} & U_{s^{n} 2} & U_{s^{n} 3} & \cdot & \cdot & U_{s^{n}(n+3)}
\end{array}\right) \\
& \approx\left(\begin{array}{ll}
N_{3 \times 3} & \Theta_{3 \times n} \\
R_{n \times 3} & S_{n \times n}
\end{array}\right) \quad \text { with } \quad \mathcal{U U}^{\dagger}=I,
\end{aligned}
$$

where $N_{3 \times 3}$ is the active neutrino mixing matrix which is no more unitary, $\Theta_{3 \times n}$ and $R_{n \times 3}$ are active-sterile neutrino mixing matrices, and $S_{n \times n}$ is sterile-sterile neutrino mixing matrix. It should be also noted that the submatrix $\left.\mathcal{W}\left(=\left[\begin{array}{ll}N & \Theta\end{array}\right]\right)\right)$ of $\mathcal{U}$ satisfy the unitarity relation

$$
\mathcal{W} \mathcal{W}^{\dagger}=N N^{\dagger}+\Theta \Theta^{\dagger}=I .
$$

Generally, the nonunitary active neutrino mixing matrix $N$ is decomposed in two ways:

(i) In terms of $\eta\left(=\frac{1}{2} \Theta^{\dagger} \Theta\right)$ parameters

$$
N=(1-\eta) U_{\mathrm{PMNS}}
$$

(ii) In terms of lower triangular matrix $\mathrm{T}$ with parameter $\alpha$,

$$
N=T U=(I-\alpha) U
$$

where $U$ is the standard neutrino mixing matrix. The explicit form of triangular matrix $T$ is given by

$$
T=\left(\begin{array}{ccc}
\alpha_{11} & 0 & 0 \\
\alpha_{21} & \alpha_{22} & 0 \\
\alpha_{31} & \alpha_{32} & \alpha_{33}
\end{array}\right)
$$

where the diagonal elements of $T$ are of the form $\left(1-\alpha_{i i}\right) \rightarrow \alpha_{i i}$.

The relation between these two parametrizations of nonunitary mixing is derived in [31] and is given by

$$
\left(\begin{array}{ccc}
\eta_{11} & 0 & 0 \\
2 \eta_{12}^{*} & \eta_{22} & 0 \\
2 \eta_{13}^{*} & 2 \eta_{23}^{*} & \eta_{33}
\end{array}\right)=\left(\begin{array}{ccc}
\alpha_{11} & 0 & 0 \\
\alpha_{21} & \alpha_{22} & 0 \\
\alpha_{31} & \alpha_{32} & \alpha_{33}
\end{array}\right) .
$$

This paper follows the parametrization of $N$ in terms of triangular matrix as it is the preferred one for oscillation studies. In presence of nonunitary mixing the flavor state of neutrino can be written as 


$$
\left|\nu_{\alpha}\right\rangle=\sum_{i} N_{\alpha i}\left|\nu_{i}\right\rangle
$$

As neutrino propagates the mass eigenstate evolves as

$$
i \frac{d}{d t}\left|\nu_{i}\right\rangle=\mathcal{H}_{0}\left|\nu_{i}\right\rangle
$$

where $\mathcal{H}_{0}$ is a Hamiltonian in vacuum, i.e.,

$$
\mathcal{H}_{0}=\frac{1}{2 E}\left(\begin{array}{ccc}
0 & 0 & 0 \\
0 & \Delta m_{21}^{2} & 0 \\
0 & 0 & \Delta m_{31}^{2}
\end{array}\right)
$$

The nonunitary neutrino oscillation probability in vacuum is given by

$$
\begin{aligned}
P\left(\nu_{\mu} \rightarrow \nu_{e}\right)= & \sum_{i, j}^{3} N_{\mu i}^{*} N_{e i} N_{\mu j} N_{e j}^{*} \\
& -4 \sum_{j>i}^{3} \operatorname{Re}\left[N_{\mu j} N_{e j} N_{\mu i} N_{e i}^{*}\right] \sin ^{2}\left(\frac{\Delta m_{j i}^{2} L}{4 E}\right) \\
& +2 \sum_{j>i}^{3} \operatorname{Im}\left[N_{\mu j}^{*} N_{e j} N_{\mu i} N_{e i}\right] \sin \left(\frac{\Delta m_{j i}^{2} L}{2 E}\right) .
\end{aligned}
$$

And the explicit form by neglecting cubic products of $\alpha_{21}$, $\sin \theta_{13}$, and $\Delta m_{21}^{2}$ gives [32]

$$
P\left(\nu_{\mu} \rightarrow \nu_{e}\right)=\left(\alpha_{11} \alpha_{22}\right)^{2} P_{\mu e}^{S O}+\alpha_{11}^{2} \alpha_{22}\left|\alpha_{21}\right| P_{\mu e}^{I}+\alpha_{11}^{2}\left|\alpha_{21}\right|^{2},
$$

where $P_{\mu e}^{S O}$ is the vacuum neutrino oscillation probability in standard three flavor oscillation framework, i.e.,

$$
\begin{aligned}
P_{\mu e}^{S O}= & \sin 2 \theta_{12} \cos ^{2} \theta_{23} \sin ^{2}\left(\frac{\Delta m_{21}^{2} L}{4 E}\right) \\
& +\sin 2 \theta_{13} \sin ^{2} \theta_{23} \sin ^{2}\left(\frac{\Delta m_{31}^{2} L}{4 E}\right) \\
& +\sin 2 \theta_{12} \sin 2 \theta_{23} \sin \theta_{13} \sin \left(\frac{\Delta m_{21}^{2} L}{2 E}\right) \\
& \times \sin \left(\frac{\Delta m_{31}^{2} L}{4 E}\right) \cos \left(\frac{\Delta m_{31}^{2} L}{4 E}-I_{123}\right),
\end{aligned}
$$

and $P_{\mu e}^{I}$ is the term which contain the new phase and explicit form is given by

$$
\begin{aligned}
P_{\mu e}^{I}= & -2\left[\sin 2 \theta_{13} \sin \theta_{23} \sin \left(\frac{\Delta m_{31}^{2} L}{4 E}+I_{N P}-I_{123}\right)\right] \\
& -\cos \theta_{13} \cos \theta_{23} \sin 2 \theta_{12} \sin \left(\frac{\Delta m_{21}^{2} L}{2 E}\right) \sin \left(I_{N P}\right),
\end{aligned}
$$

with $I_{123}=-\delta_{C P}=\phi_{12}-\phi_{13}+\phi_{23}$ and $I_{N P}=\phi_{12}-$ $\operatorname{Arg}\left(\alpha_{21}\right)$. The propagation of neutrino through matter is governed by the charged current $\left(V_{\mathrm{CC}}=\sqrt{2} G_{F} n_{e}\right)$ and neutral current $\left(V_{\mathrm{NC}}=-G_{F} n_{n} / \sqrt{2}\right)$ matter potentials. In presence of nonunitary neutrino mixing, the charged current (CC) and neutral current (NC) interaction Lagrangian becomes [22]

$$
\begin{aligned}
-\mathcal{L}_{\text {int }}= & V_{\mathrm{CC}} \sum_{i, j} N_{e i}^{*} N_{e j} \bar{\nu}_{i} \gamma^{0} \nu_{j} \\
& +V_{\mathrm{NC}} \sum_{\alpha, i, j} N_{\alpha i}^{*} N_{\alpha j} \bar{\nu}_{i} \gamma^{0} \nu_{j},
\end{aligned}
$$

which yields the effective Hamiltonian as

$$
\begin{aligned}
\mathcal{H}_{m}^{N}= & \frac{1}{2 E}\left(\begin{array}{ccc}
0 & 0 & 0 \\
0 & \Delta m_{21}^{2} & 0 \\
0 & 0 & \Delta m_{31}^{2}
\end{array}\right) \\
& +N^{\dagger}\left(\begin{array}{ccc}
V_{\mathrm{CC}}+V_{\mathrm{NC}} & 0 & 0 \\
0 & V_{\mathrm{NC}} & 0 \\
0 & 0 & V_{\mathrm{NC}}
\end{array}\right) N
\end{aligned}
$$

Then the nonunitary oscillation probability after traveling a distance $L$ yields

$$
P_{\alpha \beta}(E, L)=\mid\left\langle\nu_{\beta}\left|\nu_{\alpha}(L)\right|^{2}=\left|\left(N e^{-i \mathcal{H}_{m}^{N} L} N^{\dagger}\right)_{\beta \alpha}\right|^{2} .\right.
$$

An attempt to obtain the explicit analytical expression for neutrino oscillation probability in presence of nonunitary mixing is done in [45]. Further, an explicit perturbative calculation up to the first order in the $\nu_{\mu} \rightarrow \nu_{e}$ oscillation channel has been done in $[45,46]$. However, in this paper numerical calculations is done by using the General Long Baseline Experiment Simulator (GLoBES) $[47,48]$ package along with the plugin MonteCUBES [49]. The neutrino oscillation parameters that are considered in this analysis are given in the Table I. From the previous analysis on nonunitary parameters $[50,51]$, it is identified that the parameters in the 21 sector plays major role in $\nu_{\mu}$ to $\nu_{e}$ oscillation channel. Therefore, now onwards, the discussion is focused on nonunitary parameters in the 21 sector, i.e., $\alpha_{21}$ and its corresponding new phase $\phi_{21}$. Though the

TABLE I. The values of neutrino oscillation parameters used in the analysis [52].

\begin{tabular}{lcccccr}
\hline \hline Parameters & $\sin ^{2} \theta_{12}$ & $\sin ^{2} 2 \theta_{13}$ & $\sin ^{2} \theta_{23}$ & $\Delta m_{21}^{2}$ & $\Delta m_{\text {atm }}^{2} \mathrm{NH}(\mathrm{IH})$ & $\delta_{C P}$ \\
\hline Best fit & 0.307 & 0.085 & 0.5 & $7.4 \times 10^{-5} \mathrm{eV}^{2}$ & $2.5(-2.4) \times 10^{-3} \mathrm{eV}^{2}$ & $-90^{\circ}$ \\
\hline \hline
\end{tabular}




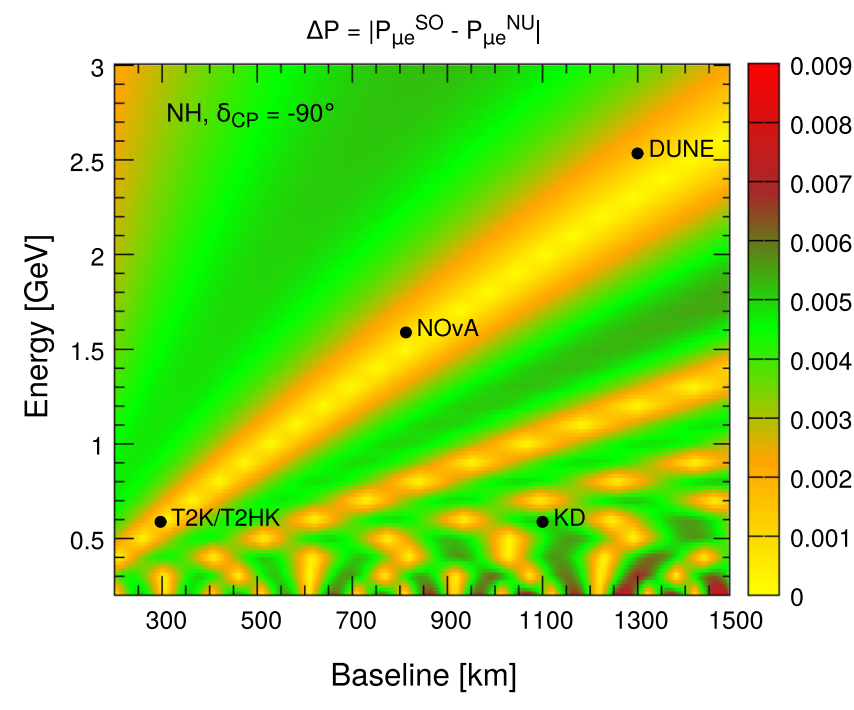

FIG. 1. $\Delta P_{\mu e}$ in $L-E$ plane for the nonunitarity parameter $\alpha_{21}=0.01$. The mass hierarchy of neutrino is assumed to be normal and other oscillation parameters are taken as given in Table I.

phase associated with the complex nonunitarity parameter can take values from $-\pi$ to $\pi$, the new phase is assumed to be zero while doing the analysis unless otherwise mentioned.

To quantify the deviation from unitary mixing of neutrinos, one can define $\Delta P_{\mu e}=\left|P_{\mu e}^{N U}-P_{\mu e}^{S O}\right|$, where $P_{\mu e}^{N U}$ and $P_{\mu e}^{S O}$, respectively, are the oscillation probability in the nonunitary and unitary mixing schemes. As the LBL experiments are mainly searching for $\nu_{\mu} \rightarrow \nu_{e}$ and $\bar{\nu}_{\mu} \rightarrow \bar{\nu}_{e}$ oscillation signals, the relative deviation in the $\nu_{\mu} \rightarrow \nu_{e}$ oscillation probability due to the nonunitary mixing as a function of all possible neutrino energies and baselines is shown in Fig. 1. The color gradient corresponds to relative deviation in oscillation probability. From the figure, it can be seen that the nonunitary parameters in the 21 sector can be probed at LBL experiments like $\mathrm{T} 2 \mathrm{~K}, \mathrm{NO} \nu \mathrm{A}, \mathrm{T} 2 \mathrm{HK}$, T2HKK, and DUNE.

\section{SIMULATION DETAILS}

This section briefly describes the experimental features of the LBL experiments at J-PARC: T2K, T2HK, and T2HKK, which are considered in this analysis.

T2K $[53,54]$ is a currently running LBL experiment which has already started collecting data from 2010 onwards, whereas T2HK and T2HKK are the proposed experiments which are considered as the upgraded version of T2K experiment. T2K experiment completed its scheduled run and now it is upgraded to phase II and continuing to take neutrino data. The muon neutrino/antineutrino beam for all these experiments is produced in the JPARC accelerator facility at Tokai. However, the WaterCerenkov detectors of these experiments are located at different locations. The detectors of $\mathrm{T} 2 \mathrm{~K}$ and $\mathrm{T} 2 \mathrm{HK}$ experiments are kept at $295 \mathrm{~km}$ away from the neutrino beam source. T2HKK experiment has two detectors: the first detector so-called Japan Detector (JD), which plans to keep $295 \mathrm{~km}$ away from the source at Japan, and the second so-called Korean Detector (KD) plans to keep at Korea about $1100 \mathrm{~km}$ away from the source. Moreover, the fiducial mass of detectors of each experiments are different. The detector fiducial mass of T2K experiment is $22.5 \mathrm{kt}$. Initial plan of $\mathrm{T} 2 \mathrm{HK}$ experiment is to consider $560 \mathrm{kt}$ fiducial mass for the detector. However, the recent plan of this experiment is to consider $374 \mathrm{kt}$. Each of the two detectors of the T2HKK experiment (JD and KD) is having a fiducial mass of $187 \mathrm{kt}$. The detector of T2HK is also known as $2 \mathrm{JD}$ as the fiducial mass of T2HK detector is twice that of JD detector of T2HKK. The detector of all these experiments is kept at an off-axis angle $2.5^{\circ}$ to the neutrino beam line which helps the neutrino flux to peak sharply at first (second) oscillation maximum of $0.6 \mathrm{GeV}$ for detector, which is kept at Japan (Korea). Further, such off-axis beam nature also reduces the intrinsic $\nu_{e}$ contamination in the beam and the background due to neutral current events and thus helps to improve the signal-tobackground ratio by a great extent.

A proton beam power of $750 \mathrm{~kW}$ and with a proton energy of $30 \mathrm{GeV}$, which corresponds to a total exposure of $7.8 \times 10^{21}$ protons on target (POT) with a $1: 1$ ratio of neutrino to antineutrino modes, is considered to simulate T2K experiment for this study [55]. The signal and background event spectra and rates are matched with those given in the recent publication of the T2K collaboration [56]. An uncorrelated 5\% normalization error on signal and $10 \%$ normalization error on background for both the appearance and disappearance channels are considered as given in [56] to analyze the prospective data from the T2K experiment, and it is assumed that the set of systematics for both the neutrino and antineutrino channels are uncorrelated.

A total of 10 years of operation with $1.3 \mathrm{MW}$ beam power with $1: 3$ ratio of neutrino to antineutrino modes which corresponds to $27 \times 10^{21}$ POT by following [57,58] is considered to simulate both T2HK and T2HKK experiments. Moreover, both signal and background event spectra and rates are matched with those given in [58]. An uncorrelated 5\% normalization error on signal and 10\% normalization error on background for both the appearance and disappearance channels are used as the way considered for those of the T2K experiment. For the simulations, GLoBES along with MonteCUBES have been used. Further, the Poissonian $\chi^{2}$ is evaluated using GLoBES package [59-61] and its explicit from is given by

$$
\begin{aligned}
\chi^{2}= & \min _{\xi_{l}, \vec{P}} \sum_{i=1}^{N_{E}}\left[2 N_{i}^{\mathrm{th}}(\vec{P}, \xi)-2 N_{i}^{\mathrm{dat}}(\vec{P}, \xi)\right. \\
& \left.-2 N_{i}^{\mathrm{dat}}(\vec{P}, \xi) \ln \left(\frac{N_{i}^{\mathrm{th}}(\vec{P}, \xi)}{N_{i}^{\mathrm{dat}}(\vec{P}, \xi)}\right)\right]+\sum_{l=1}^{2} \xi_{l}^{2},
\end{aligned}
$$


where $N_{i}^{\text {th }}(\vec{P}, \xi)$ and $N_{i}^{\mathrm{dat}}(\vec{P}, \xi)$, respectively, are the expected and observed events (both signal and background) for a considered $i$ th energy bin. Further, $N_{i}^{\text {th }}(\vec{P}, \xi)=$ $N_{i}^{0}\left(1+\sum_{l=1}^{2} \pi_{i}^{l} \xi_{l}^{2}\right)$ with $N_{i}^{0}$ as the number of events without systematics, $N_{E}$ is the total number of energy bins, $\xi_{1}$ and $\xi_{2}$ are the systematic errors associated with signal and background events, respectively, with $\vec{P}=\left\{\theta_{12}, \theta_{13}, \theta_{23}, \Delta \mathrm{m}_{21}^{2}\right.$, $\left.\Delta \mathrm{m}_{31}^{2}, \delta_{C P}\right\}$ representing all the fundamental oscillation parameters, while $\vec{p}=\left\{\theta_{23}, \delta_{C P}, \Delta m_{31}^{2}\right\}$ is the subset of $\vec{P}$ on which we perform marginalization. The marginalization range for $\delta_{C P}, \sin ^{2} \theta_{23}$, and $\Delta m_{31}^{2}$ are $\left[-180^{\circ}: 180^{\circ}\right]$, [0.4:0.6], and [0.36:0.64], respectively. Moreover, in this work, $\Delta \chi^{2}$ is determined using the pull variable over the systematic uncertainties and a detailed discussion on this is given in $[60,61]$.

\section{RESULTS AND DISCUSSIONS}

This section mainly discuss the capability of LBL experiments based at J-PARC in establishing unitarity of neutrino mixing matrix. Further, this section also discusses the bounds on NU parameter and sensitivity limits of LBL experiment to determine NU parameter.

As this study is focusing on the nonunitarity parameters $\alpha_{21}$ and its corresponding $C P$-violating phases, it is most important to know how these parameters affect the oscillation probability. Though it is always better to start with analysis by looking at the analytical expression of neutrino oscillation probability to have a deep understanding of physics, the oscillation probability is calculated numerically as a function of neutrino energy for this study, as is shown in Fig. 2. In the figure, the black dashed curve

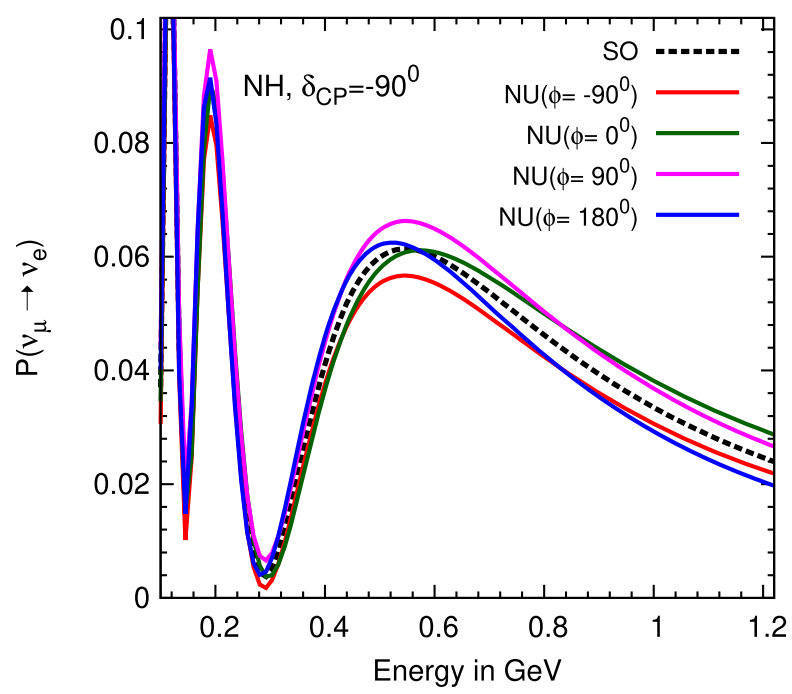

corresponds to the oscillation probability in standard oscillation case with normal hierarchy and $\delta_{C P}=-90^{\circ}$, whereas the red, green, magenta, and blue solid curves correspond to oscillation probabilities in the presence of NU parameter $\alpha_{21}=0.01$ with $\phi_{21}=-90^{\circ}, 0^{\circ}, 90^{\circ}$, and $180^{\circ}$, respectively. The left (right) panel is for oscillation probability for neutrino (antineutrino). From the figure, it can be seen that for $\phi_{21}=90^{\circ},-90^{\circ}$, there is a significant deviation from the standard oscillation case, whereas there is no significant deviation for $\phi_{21}=0^{\circ}, 180^{\circ}$. Therefore, $\phi_{21}=90^{\circ},-90^{\circ}$ are the favorable values of new phase to rule out the nonunitary mixing and $\phi_{21}=0^{\circ}, 180^{\circ}$ are the unfavorable values as there is no much deviation from standard oscillation case. It should be also noted that the oscillation curves for $\phi_{21}=0^{\circ}, 180^{\circ}$ are touching the standard oscillation curve at some point where one cannot distinguish between nonunitary mixing and unitary mixing. However, such intersection point is different in neutrino and antineutrino oscillation channels. Therefore, an interplay of neutrino and antineutrino oscillation helps in distinguishing unitary mixing from nonunitary mixing.

Now the focus will be on the unfavorable values of new phase, i.e., $\phi=0^{\circ}$, as it is challenging for this value to distinguish nonunitary mixing from unitary mixing. In order to show the capability of a LBL experiment to establish unitary mixing as a function of true values of $\delta_{C P}$, one can define

$$
\Delta \chi_{N U}^{2}=\chi_{S O}^{2}-\chi_{N U}^{2}
$$

where $\chi_{S O}^{2}$ is evaluated using Eq. (17) by assuming both $N^{\text {th }}$ and $N^{\text {dat }}$ are with unitary mixing. Whereas, $\chi_{N U}^{2}$ is calculated by assuming $N^{\text {th }}$ with nonunitary mixing and

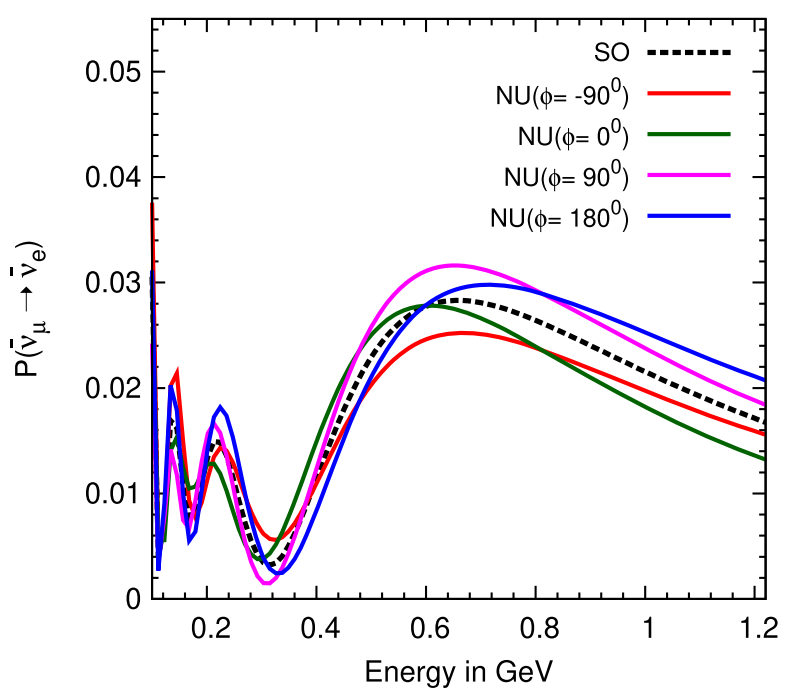

FIG. 2. The neutrino (antineutrino) oscillation probability as a function of neutrino energy for T2K/T2HK in the left (right) panel. The black dashed curve corresponds to the oscillation probability in standard oscillation paradigm with $\delta_{C P}=-90^{\circ}$, whereas the red, green, magenta, and blue solid curves correspond to oscillation probabilities in the presence of NU parameter $\alpha_{21}=0.01$ with $\phi_{21}=-90^{\circ}, 0^{\circ}, 90^{\circ}$, and $180^{\circ}$, respectively. Neutrino mass hierarchy is assumed to be normal. The left (right) panel is for oscillation probability for neutrino (antineutrino). 

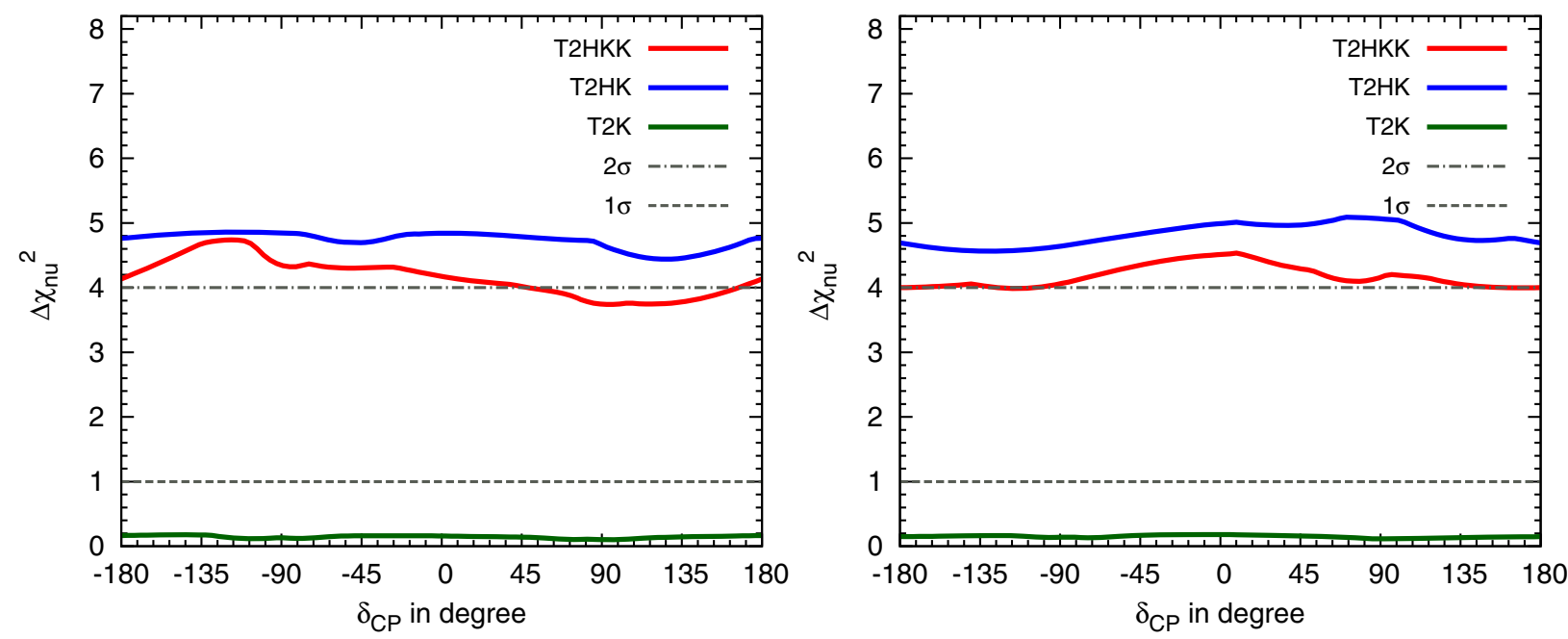

FIG. 3. The capability of LBL experiments in establishing the unitary mixing by ruling out the nonunitary mixing as a function of true values of $\delta_{C P}$. In the left (right) panel the mass hierarchy of neutrino is assumed to be normal (inverted).

$N^{\text {dat }}$ with unitary mixing. The minimum value for $\Delta \chi_{N U}^{2}$ is obtained by doing marginalization over oscillation parameters including the new phase $\phi_{21}$ in its allowed range $\left[-180^{\circ}: 180^{\circ}\right]$. The minimized $\Delta \chi_{N U}^{2}$ as a function of true values of $\delta_{C P}$ is shown in Fig. 3. In the left (right) panel of the figure, hierarchy is assumed to be normal (inverted) and the atmospheric mixing angle is set to maximal mixing. From the figure, it can be seen that the T2K experiment
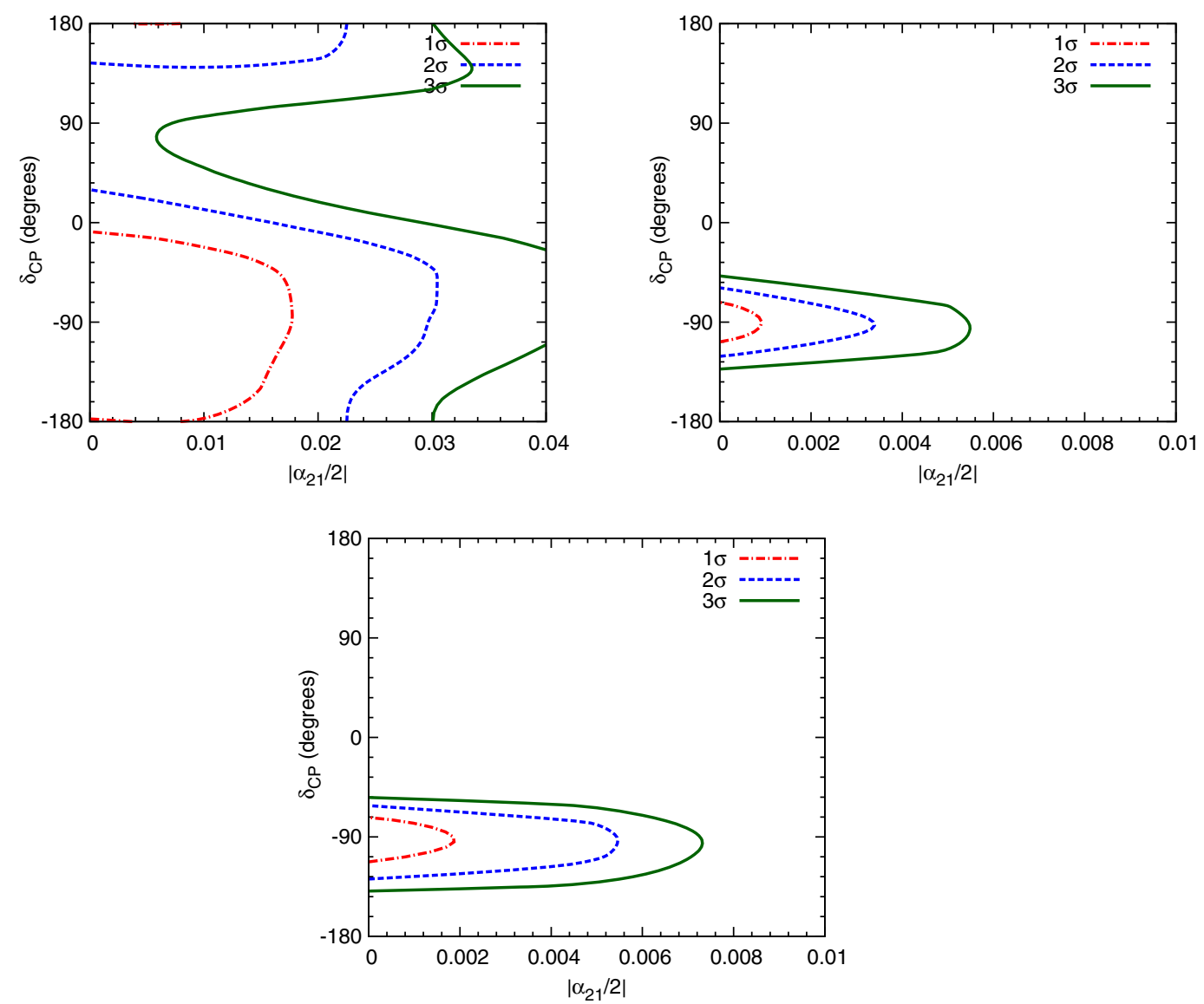

FIG. 4. The bounds on the NU parameter is obtained by comparing unitary mixing against nonunitary mixing. The upper left (right) panel is for T2K (T2HK) and lower panel is for T2HKK. The red, blue, and green curves are, respectively, for $1 \sigma, 2 \sigma$, and $3 \sigma$ C.L. contours. The neutrino mass hierarchy is assumed to be normal and other oscillation parameters are used as given in Table I. 


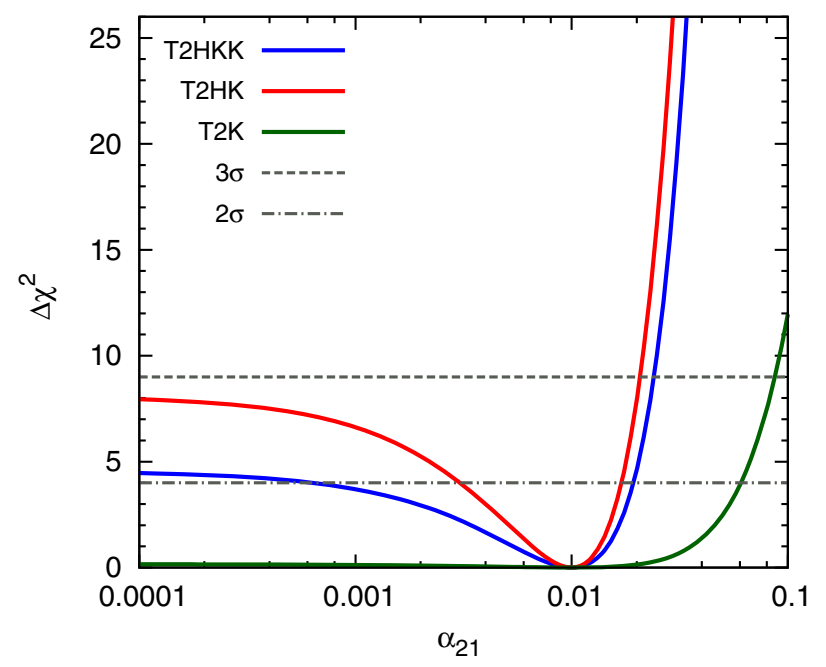

FIG. 5. The precision measurement of $\alpha_{21}$ at LBL experiments. The mass hierarchy of neutrino is assumed to be normal and other oscillation parameters are set as given in Table I.

cannot rule out the nonunitary mixing with the data so far collected. Whereas T2HKK can rule out nonunitary mixing with a significance of more than $2 \sigma$ for most of the values of $\delta_{C P}$. Moreover, $\mathrm{T} 2 \mathrm{HK}$ can rule out nonunitary mixing above $2 \sigma$ C.L. irrespective of mass hierarchy and the true value of $\delta_{C P}$. From the analysis, it is found that the interplay between neutrino and antineutrino runs helps in ruling out the nonunitary mixing.

The bounds on NU parameters using LBL experiment is obtained by comparing unitary mixing with oscillation parameters as shown in Table I against nonunitary mixing. The minimized $\Delta \chi^{2}$ is evaluated by doing marginalization over oscillation parameters and it is shown in the $\delta_{C P}^{\text {test }}-$ $\left(\alpha_{21} / 2\right)^{\text {test }}$ plane as given in Fig. 4. The red, blue, and green lines are, respectively, $1 \sigma, 2 \sigma$, and $3 \sigma$ C.L. contours. From the figure, it can be seen that the bounds from T2K experiment on NU parameter is not significantly constraint, whereas that for T2HK and T2HKK is severely constraint. Further, the bounds on $\alpha_{21} / 2$ are $0.028,0.0026,0.005$ at $2 \sigma$ C.L., respectively, for T2K, T2HK, and T2HKK.

Next, analyzing the sensitivity limit of LBL experiments in determining NU parameters. In order to obtain this, the experimental data is simulated by fixing true oscillation parameters as given in Table I and setting true value of $\alpha_{21}=0.01$, then comparing it with theory by varying $\alpha_{21}$ in the range [0.001:0.1]. Further, the $\Delta \chi^{2}$ is minimized by doing marginalization over oscillation parameters and nonunitary phase and it is given in Fig. 5. From the figure, it can be seen that the precision in the measurement of $\alpha_{21}$ by T2HK is better than that of both T2K and T2HKK.

\section{SUMMARY AND CONCLUSIONS}

The measurement of neutrino oscillation parameters in a three flavor framework is usually done by assuming that the active neutrino mixing matrix is unitary. However, the extended theories to accommodate massive neutrinos indicate the existence of new neutrino states that can give rise to the nonunitary mixing of active neutrinos. This paper mainly scrutinized whether the long baseline experiments like T2K, T2HK, and T2HKK can establish the unitarity of active neutrino matrix by ruling out such nonunitary mixing in the 21 sector. It is found that T2HK can establish unitarity of active neutrino mixing above $2 \sigma$ C.L. irrespective of neutrino mass hierarchy and true value of $\delta_{C P}$ if NU parameter $\alpha_{21}$ of the order of $10^{-2}$. Further, this paper also investigated the bound on NU parameters that can be achieved from these LBL experiments and found that the bounds on $\alpha_{21} / 2$ are $0.028,0.0026,0.005$ at $2 \sigma$ C.L., respectively, for T2K, T2HK, and T2HKK. Finally, it is also found that the sensitivity limit of T2HK on NU parameter is far better than that of both T2HKK and $\mathrm{T} 2 \mathrm{~K}$.

\section{ACKNOWLEDGMENTS}

The author would like to thank the organizers of the workshop XIX International Workshop on Neutrino Telescopes held online during February 18-26, 2021, for giving an opportunity to present the preliminary results of this work. The author also would like to thank the Science and Engineering Research Board (SERB) for the funding under National Post-Doctoral Fellowship (NPDF) scheme [Grant No. PDF/2019/003346].
[1] M. Apollonio et al. (CHOOZ Collaboration), Initial results from the $\mathrm{CHOOZ}$ long baseline reactor neutrino oscillation experiment, Phys. Lett. B 420, 397 (1998).

[2] Y. Fukuda et al. (Super-Kamiokande Collaboration), Neutrino-induced upward stopping muons in SuperKamiokande, Phys. Lett. B 467, 185 (1999).
[3] S. Fukuda et al. (Super-Kamiokande Collaboration), Tau Neutrinos Favored over Sterile Neutrinos in Atmospheric Muon Neutrino Oscillations, Phys. Rev. Lett. 85, 3999 (2000).

[4] S. Fukuda et al. (Super-Kamiokande Collaboration), Solar ${ }^{8} B$ and hep Neutrino Measurements from 1258 Days of Super-Kamiokande Data, Phys. Rev. Lett. 86, 5651 (2001). 
[5] Q. R. Ahmad et al. (SNO Collaboration), Measurement of the Rate of $\nu e+d \rightarrow p+p+e^{-}$Interactions Produced by ${ }^{8} B$ Solar Neutrinos at the Sudbury Neutrino Observatory, Phys. Rev. Lett. 87, 071301 (2001).

[6] T. Araki et al. (KamLAND Collaboration), Measurement of Neutrino Oscillation with KamLAND: Evidence of Spectral Distortion, Phys. Rev. Lett. 94, 081801 (2005).

[7] S. Abe et al. (KamLAND Collaboration), Precision Measurement of Neutrino Oscillation Parameters with KamLAND, Phys. Rev. Lett. 100, 221803 (2008).

[8] R. N. Mohapatra and G. Senjanovic, Neutrino Mass and Spontaneous Parity Violation, Phys. Rev. Lett. 44, 912 (1980).

[9] M. Magg and C. Wetterich, Neutrino mass problem and gauge hierarchy, Phys. Lett. 94B, 61 (1980).

[10] J. Schechter and J.W.F. Valle, Neutrino masses in $\mathrm{SU}(2) \times \mathrm{U}(1)$ theories, Phys. Rev. D 22, 2227 (1980).

[11] G. Lazarides, Q. Shafi, and C. Wetterich, Proton lifetime and fermion masses in an $\mathrm{SO}(10)$ model, Nucl. Phys. B181, 287 (1981).

[12] R. N. Mohapatra and G. Senjanovic, Neutrino masses and mixings in gauge models with spontaneous parity violation, Phys. Rev. D 23, 165 (1981).

[13] C. Wetterich, Neutrino masses and the scale of B-L violation, Nucl. Phys. B187, 343 (1981).

[14] E. Ma, Pathways to Naturally Small Neutrino Masses, Phys. Rev. Lett. 81, 1171 (1998).

[15] E. Ma and D. P. Roy, Heavy triplet leptons and new gauge boson, Nucl. Phys. B644, 290 (2002).

[16] T. Hambye, Y. Lin, A. Notari, M. Papucci, and A. Strumia, Constraints on neutrino masses from leptogenesis models, Nucl. Phys. B695, 169 (2004).

[17] R. N. Mohapatra and J. W. F. Valle, Neutrino mass and baryon-number nonconservation in superstring models, Phys. Rev. D 34, 1642 (1986).

[18] D. V. Forero, S. Morisi, M. Tortola, and J. W. F. Valle, Lepton flavor violation and non-unitary lepton mixing in low-scale type-I seesaw, J. High Energy Phys. 09 (2011) 142.

[19] J. Schechter and J. W. F. Valle, Neutrino decay and spontaneous violation of lepton number, Phys. Rev. D 25, 774 (1982).

[20] A. Ibarra and G. G. Ross, Neutrino phenomenology: The case of two right-handed neutrinos, Phys. Lett. B 591, 285 (2004).

[21] M. Hirsch, S. Morisi, and J. W. F. Valle, A4-based tribimaximal mixing within inverse and linear seesaw schemes, Phys. Lett. B 679, 454 (2009).

[22] S. Antusch, C. Biggio, E. Fernandez-Martinez, M. B. Gavela, and J. Lopez-Pavon, Unitarity of the leptonic mixing matrix, J. High Energy Phys. 10 (2006) 084.

[23] S. Goswami and T. Ota, Testing non-unitarity of neutrino mixing matrices at neutrino factories, Phys. Rev. D 78, 033012 (2008).

[24] P. S. B. Dev and R. N. Mohapatra, TeV scale inverse seesaw in $\mathrm{SO}(10)$ and leptonic non-unitarity effects, Phys. Rev. D 81, 013001 (2010).

[25] A. Abada, D. Das, A. M. Teixeira, A. Vicente, and C. Weiland, Tree-level lepton universality violation in the presence of sterile neutrinos: Impact for $R_{K}$ and $R_{\pi}$, J. High Energy Phys. 02 (2013) 048.
[26] A. Abada, A. M. Teixeira, A. Vicente, and C. Weiland, Sterile neutrinos in leptonic and semileptonic decays, J. High Energy Phys. 02 (2014) 091.

[27] A. Abada, M. E. Krauss, W. Porod, F. Staub, A. Vicente, and C. Weiland, Lepton flavor violation in low-scale seesaw models: SUSY and non-SUSY contributions, J. High Energy Phys. 11 (2014) 048.

[28] R. L. Awasthi, M. K. Parida, and S. Patra, Neutrino masses, dominant neutrinoless double beta decay, and observable lepton flavor violation in left-right models and $\mathrm{SO}(10)$ grand unification with low mass $W_{R}, Z_{R}$ bosons, J. High Energy Phys. 08 (2013) 122.

[29] S. Emelyanov, Non-unitarity or hidden observables?, arXiv: 1410.6149.

[30] S. Antusch and O. Fischer, Probing the nonunitarity of the leptonic mixing matrix at the CEPC, Int. J. Mod. Phys. A 31, 1644006 (2016).

[31] M. Blennow, P. Coloma, E. Fernandez-Martinez, J. Hernandez-Garcia, and J. Lopez-Pavon, Non-unitarity, sterile neutrinos, and non-standard neutrino interactions, J. High Energy Phys. 04 (2017) 153.

[32] F. J. Escrihuela, D. V. Forero, O. G. Miranda, M. Tortola, and J. W. F. Valle, On the description of nonunitary neutrino mixing, Phys. Rev. D 92, 053009 (2015); 93, 119905(E) (2016).

[33] M. Malinsky, T. Ohlsson, Z.z. Xing, and H. Zhang, Non-unitary neutrino mixing and $C P$ violation in the minimal inverse seesaw model, Phys. Lett. B 679, 242 (2009).

[34] S. Antusch, J. P. Baumann, and E. Fernandez-Martinez, Non-standard neutrino interactions with matter from physics beyond the standard model, Nucl. Phys. B810, 369 (2009).

[35] S. Antusch and O. Fischer, Non-unitarity of the leptonic mixing matrix: Present bounds and future sensitivities, J. High Energy Phys. 10 (2014) 094.

[36] F. J. Escrihuela, D. V. Forero, O. G. Miranda, M. Trtola, and J. W. F. Valle, Probing $C P$ violation with non-unitary mixing in long-baseline neutrino oscillation experiments: DUNE as a case study, New J. Phys. 19, 093005 (2017).

[37] D. Dutta, P. Ghoshal, and S. Roy, Effect of non unitarity on neutrino mass hierarchy determination at DUNE, $\mathrm{NO} \nu \mathrm{A}$ and T2K, Nucl. Phys. B920, 385 (2017).

[38] D. Dutta and P. Ghoshal, Probing $C P$ violation with T2K, $\mathrm{NO} \nu \mathrm{A}$ and DUNE in the presence of non-unitarity, J. High Energy Phys. 09 (2016) 110.

[39] S. Verma and S. Bhardwaj, Probing non-unitary $C P$ violation effects in neutrino oscillation experiments, Indian J. Phys. 92, 1161 (2018).

[40] D. V. Forero, C. Giunti, C. A. Ternes, and M. Tórtola, Nonunitary neutrino mixing in short and long-baseline experiments, Phys. Rev. D 104, 075030 (2021).

[41] Z. Hu, J. Ling, J. Tang, and T. Wang, Global oscillation data analysis on the $3 \nu$ mixing without unitarity, J. High Energy Phys. 01 (2021) 124.

[42] S. A. R. Ellis, K. J. Kelly, and S. W. Li, Current and future neutrino oscillation constraints on leptonic unitarity, J. High Energy Phys. 12 (2020) 068. 
[43] L. S. Miranda, P. Pasquini, U. Rahaman, and S. Razzaque, Searching for non-unitary neutrino oscillations in the present T2K and NO $\nu$ A data, Eur. Phys. J. C 81, 444 (2021).

[44] F. J. Escrihuela, L. J. Flores, and O. G. Miranda, Neutrino counting experiments and non-unitarity from LEP and future experiments, Phys. Lett. B 802, 135241 (2020).

[45] C. S. Fong, H. Minakata, and H. Nunokawa, Non-unitary evolution of neutrinos in matter and the leptonic unitarity test, J. High Energy Phys. 02 (2019) 015.

[46] I. Martinez-Soler and H. Minakata, Physics of parameter correlations around the solar-scale enhancement in neutrino theory with unitarity violation, Prog. Theor. Exp. Phys. 2020, 113B01 (2020).

[47] P. Huber, M. Lindner, and W. Winter, From parameter space constraints to the precision determination of the leptonic Dirac CP phase, J. High Energy Phys. 05 (2005) 020.

[48] P. Huber, M. Lindner, T. Schwetz, and W. Winter, First hint for $C P$ violation in neutrino oscillations from upcoming superbeam and reactor experiments, J. High Energy Phys. 11 (2009) 044.

[49] M. Blennow and E. Fernandez-Martinez, Neutrino oscillation parameter sampling with MonteCUBES, Comput. Phys. Commun. 181, 227 (2010).

[50] C. Soumya and M. Rukmani, Non-unitary lepton mixing in an inverse seesaw and its impact on the physics potential of long-baseline experiments, J. Phys. G 45, 095003 (2018).

[51] S. C and R. Mohanta, Non-unitary lepton mixing in an inverse seesaw and its impact on the physics potential of long-baseline experiments, arXiv:1708.05372.

[52] I. Esteban, M. C. Gonzalez-Garcia, A. HernandezCabezudo, M. Maltoni, and T. Schwetz, Global analysis of three-flavour neutrino oscillations: Synergies and tensions in the determination of $\theta_{23}, \delta_{C P}$, and the mass ordering, J. High Energy Phys. 01 (2019) 106; NuFIT 4.0, www.nu-fit.org (2018).

[53] Y. Itow et al. (T2K Collaboration), The JHF-Kamioka neutrino project, arXiv:hep-ex/0106019.

[54] M. Ishitsuka, T. Kajita, H. Minakata, and H. Nunokawa, Resolving neutrino mass hierarchy and $C P$ degeneracy by two identical detectors with different baselines, Phys. Rev. D 72, 033003 (2005).

[55] K. Abe et al. (T2K Collaboration), Combined Analysis of Neutrino and Antineutrino Oscillations at T2K, Phys. Rev. Lett. 118, 151801 (2017).

[56] K. Abe et al. (T2K Collaboration), Neutrino oscillation physics potential of the T2K experiment, Prog. Theor. Exp. Phys. 2015, $043 \mathrm{C} 01$ (2015).

[57] K. Abe et al. (Hyper-Kamiokande Collaboration), Physics potentials with the second Hyper-Kamiokande detector in Korea, Prog. Theor. Exp. Phys. 2018, 063 C01 (2018).

[58] K. Abe et al. (Hyper-Kamiokande Collaboration), HyperKamiokande design report, arXiv:1805.04163.

[59] M. C. Gonzalez-Garcia and M. Maltoni, Status of global analysis of neutrino oscillation data, in Neutrino oscillations and their origin, in Proceedings of the 5th International Workshop, NOON2004, Tokyo, Japan, 2004 (World Scientic Publishing Co Pte Ltd, 2004), p. 115124, 10.1142/9789812701824_0015.

[60] G. L. Fogli, E. Lisi, A. Marrone, D. Montanino, and A. Palazzo, Getting the most from the statistical analysis of solar neutrino oscillations, Phys. Rev. D 66, 053010 (2002).

[61] P. Huber, M. Lindner, and W. Winter, Superbeams versus neutrino factories, Nucl. Phys. B645, 3 (2002). 